\title{
Time to Drive the Change! Challenges and Opportunities in Pediatric Asthma
}

\author{
Naveen Pillarisetti ${ }^{1}$ Sushil K. Kabra ${ }^{2}$
}

Published online: 22 December 2021

(c) Dr. K C Chaudhuri Foundation 2021

Asthma, a condition of disordered inflammation and airway hyperreactivity continues to be a major cause of morbidity and mortality in children and young adults across the world. While the incidence of asthma varies widely between countries, some of these differences could be due to under-reporting, misdiagnosis, stigma surrounding diagnosis, and lack of large-scale prevalence studies, especially in the developing world $[1,2]$.

In this issue of the journal, part 1 of the special symposium on asthma covering various aspects is being published. While the treatment of asthma in the developed world has rapidly advanced to next-generation inhaled therapies and use of biological agents, the developing world is still playing catch-up. However, Prof. Bush sets the record straight and emphasizes that asthma management in any clinical setting, including the seemingly difficult patients, comes down to the basics of meticulous history-taking, physical examination, and assessment of clinical symptoms. The most important aspect is ensuring adherence to treatments using age-appropriate inhaler therapies and devices [3].

Dhochak et al. describe the burden of asthma in the developing world where the prevalence may appear less than developed countries, but the presentations may be more severe [4]. The challenges of asthma care in developing countries, such as shortage of trained man-power, lack of infrastructure, and the prohibitive cost of treatments are discussed. The stigma of asthma diagnosis and the proliferation of alternative remedies need to be challenged. This review discusses solutions to some of the problems in particular training and empowering health professionals.

Naveen Pillarisetti

naveenp@adhb.govt.nz

1 Department of Pediatric Respiratory Medicine, Starship Children's Hospital, Auckland 1023, New Zealand

2 Department of Pediatrics, All India Institute of Medical Sciences, New Delhi, India
Chronic asthma is often associated with dysfunctional breathing pattern, heightened sympathetic tone, anxiety, and depression. While medical management focuses on reducing airway inflammation and bronchodilatation, it is important for the astute clinician to recognize and address these problems and specifically, aspects of breathing which can complement standard medical care. The article by Das et al. elegantly discusses some of the breathing related pathophysiology in general, and specifically, the role of "Yoga and Pranayama" in the management of asthma [5].

The COVID-19 pandemic has had widespread socioeconomic repercussions across the world. The effects on health care delivery have been felt in most countries, both developed and developing. Routine and emergency medical care have been disrupted by multiple waves of the pandemic and emergence of new strains of the virus. Klouda et al. discuss the effect of COVID-19 pandemic on pediatric asthma [6]. Evidence suggests that this far, children in general, and children with asthma in particular, are relatively spared from the severe effects of SARS-CoV-2 virus. Despite this, there are other significant disruptive effects on delivery of chronic and acute asthma care. Disruptive technology and innovations in health care, especially the role of telehealth have helped in reaching out to families and managing children with asthma. There is no reason why we cannot refine and adopt some of these models of care even when the pandemic is over.

Experts from across the world have collaborated to bring together a series of very interesting and useful review articles on aspects of pediatric asthma in this asthma symposium.

While acknowledging the fact that there are unique challenges in the developing world, Prof. Bush calls out to the professionals in the developing world to be innovative and "drive the change" to improve the management of pediatric asthma. This appears to be the right time to call for more investments in developing infrastructure, education, training and research opportunities in pediatric respiratory medicine and asthma in the developing countries. The solution lies within. 


\section{Declarations}

Conflict of Interest None.

\section{References}

1. Lai CK, Beasley R, Crane J, Foliaki S, Shah J, Weiland S; International Study of Asthma and Allergies in Childhood Phase Three Study Group. Global variation in the prevalence and severity of asthma symptoms: phase three of the international study of asthma and allergies in childhood (ISAAC). Thorax. 2009;64:476-83.

2. Asher MI, Rutter CE, Bissell K et al; Global Asthma Network Phase I Study Group. Worldwide trends in the burden of asthma symptoms in school-aged children: Global asthma network phase I cross-sectional study. Lancet. 2021;398:1569-80.
3. Bush A. Severe and difficult asthma: diagnosis and managementchallenges for a low-resource environment. Indian J Pediatr. 2021. https://doi.org/10.1007/s12098-021-03952-w.

4. Dhochak N, Kabra SK. Challenges in the management of childhood asthma in the developing world. Indian J Pediatr. 2021. https://doi.org/10.1007/s12098-021-03941-z.

5. Das RR, Sankar J, Kabra SK. Role of breathing exercises in Asthma-Yoga and Pranayama. Indian J Pediatr. 2021. https://doi. org/10.1007/s12098-021-03998-w.

6. Klouda T, Pillarisetti A, Xie A, Kabra S, Saradhi N, Katwa U. Asthma management in the era of the COVID-19 pandemic. Indian J Pediatr. 2021. https://doi.org/10.1007/s12098-021-03979-z.

Publisher's Note Springer Nature remains neutral with regard to jurisdictional claims in published maps and institutional affiliations. 